\title{
Using Capacitance Measurements to Study Polarization in Mercuric Iodide Radiation Detectors
}

\author{
M. A. Hassan \\ Electronics Engineering Department, Princess Sumaya University, \\ Amman- Jordan
}

\begin{abstract}
Mercuric iodide is an interesting material for room temperature X-ray or gamma-ray spectrometers. This is due to its wide band gap $(2.13 \mathrm{eV})$ and its high atomic numbers (80/53). A spectral resolution of better than $4 \%$ at ${ }^{137}$ Cs has been reported. Mercuric iodide detectors suffer from polarization i.e., their electrical properties change with time and it will affect and reduce initial high spectral resolution and other electrical properties. This research paper is studying the polarization phenomena using capacitance measurements with low bias voltages and assuming a metal insulator semiconductor (MIS) structure.
\end{abstract}

\section{Introduction}

Mercuric iodide radiation detectors can operate with good resolution results at room temperature without cooling. A spectral resolution, better than $4 \%$ at ${ }^{137} \mathrm{Cs}$, has been reported [1]. This is due to their wide band gaps $(2.13 \mathrm{eV})$ that tend to reduce their thermal leakage currents. Also, the high atomic numbers, (53) for iodine and (80) for mercury, significantly increase their photoelectric absorption over traditional semiconductor materials such as silicon or germanium. Phase deposition using vertical or horizontal ampoule methods have been used to grow mercuric iodide crystals. Most of the reported growth processes were under vacuum [2,3], recent group [4] is planning to grow mercuric iodide crystals under gases such as argon or nitrogen.

Results have been obtained from detectors, which were grown by dynamic sublimation technique [5] and by temperature oscillation method [6]. Both methods showed similar results. High quality commercial mercuric iodide was used as a starting material. Several purification runs were performed on the starting material using repeated sublimations. 
The three main crystal growth difficulties of mercuric iodide crystals are: the impurities, the ampoules thermal profiles during the growth process and the vibrations. So, the reduction and the knowledge of impurities are of importance. Searching for ideal thermal profiles during crystal growth and eliminating vibration sources are needed steps.

Impurities were reduced by the repeated sublimations. After each purification run, black residue was noticed and it was reduced after subsequent run. Until now, exact knowledge of mercuric iodide impurities and their quantities are not definite due to the active and poisonous nature of mercuric iodide. Some researchers [7] have reported about the existence of hydrocarbon impurities.

The optimal crystal growth thermal profiles are important, initially to produce a suitable single nucleus and later to reach a suitable thermal profile during crystal growth period in order to have crystal uniformity and reduce crystal mechanical structural defects.

Because mercuric iodide crystal growth rate is few millimeters per day, mechanical stability of crystal growth setup is extremely important to produce crystals with less structural damage. The best growth results were achieved during holidays because vibrations produced by cars movements on nearby streets were minimal.

Very few electrical contacts options are available for mercuric iodide crystals because mercuric iodide reacts with most of the metals. Palladium contacts or colloidal carbon have been used. Also, a thin polymer coating has been used to stop any sublimation or contamination.

Mercuric iodide detectors crystals suffer from polarization effects [8-10]. Detectors performance changes and deteriorates with time. The external bias develops internal fields in such a direction to reduce the detector charge collection efficiency.

The origin and the reasons of the polarization effects are probably impurities, defect centers due to internal mechanical damage (results from nonuniform thermal profiles during crystal growth or due to vibration) or due to the continual chemical reaction of mercuric iodide with existing impurities in the bulk detector or near the surface region. The chemical reaction statement is supported by some detectors with initial good results but their performance deteriorated quickly with time. Also, polarization effect is related to the trapping of carriers in the bulk material. Trapping leads to changes in the electric field profile which will reduce the charge collection efficiency of the detector. 
In summary, polarization reasons are related to many factors such as crystal growth techniques and procedures, quality of the starting material, crystal orientation/fabrication and contact materials used.

\section{Capacitance Voltage Measurements:}

Reported capacitance-voltage $(\mathrm{C}-\mathrm{V})$ measurements for radiation detectors have been related to trapping centers and to bulk impurities $[11 \&$ 12]. Good spectroscopic mercuric iodide detectors show less than $2 \mathrm{pF}$ capacitance [13] and usually uniform in nature with applied bias voltages. Low quality mercuric iodide detectors showed high capacitance values and nonuniform with applied bias [14].

During measurements, the following figures were adapted to differentiate between good quality detectors and low quality detectors:

\begin{tabular}{|c|c|}
\hline Detector Quality & FWHM for ${ }^{55} \mathrm{Fe}(\mathrm{eV})$ \\
\hline Good & $<640$ \\
\hline Low & $>1000$ \\
\hline
\end{tabular}

Until now very little is known about the mercuric iodide crystals doping effects. Also, the internal structure of the crystals is not fully understood.

The mercuric iodide detectors could not be fully classified as MIS (metal insulator semiconductor) devices or metal semiconductor devices may be a combination of both. The following cases complicate the classifications:

- Uncovered mercuric iodide detectors react with air and establish a thin insulator cover.

- Some mercuric iodide detectors have a polymer coating to ensure long term stability. This arrangement is very close to MIS structure.

- The palladium contacts do not cover the whole surface of the crystal.

- With the colloidal carbon contacts, suitable type of polymer is needed to fix the terminal wires.

Also, it is not possible to consider a mercuric iodide detector as a silicon diode because its bulk material is not well known and its electrical current does not change with positive or negative bias voltages. But its capacitance measurement with voltage shows a peak similar to MIS devices.

This research paper is concentrating on $\mathrm{C}-\mathrm{V}$ measurements for mercuric iodide detectors to observe and study their polarization phenomena. 


\section{Results of C-V Measurements:}

HP LCR meter 4263B was used to measure the capacitances of the detectors under different bias voltages and different frequencies.

Capacitance measurements were performed on stabilized detectors, i.e. detectors which were kept in dark for at least 4 hours and capacitance measurements were immediately taken after applying bias voltages.

The $\mathrm{C}-\mathrm{V}$ measurements were taken for different detectors using colloidal carbon contacts fixed with polymer glue. The measurements showed different forms of polarization which can be summarized as follows:

- Capacitance results for polarized detectors were not consistent with time.

- Capacitance results for polarized detectors tend to decrease if detector was kept in dark for more than four hours.

- Capacitance results for polarized detectors tend to decrease if detector was subjected to strong light.

Figures $(1 \& 2)$ show $\mathrm{C}-\mathrm{V}$ measurements for a polarized detector in the range of $0-9 \mathrm{~V}$ and at $1 \mathrm{kHz} / 120 \mathrm{~Hz}$. If the detector was kept in dark for more than four hours or if it was subjected to strong light its capacitance was reduced by $10-15 \%$.

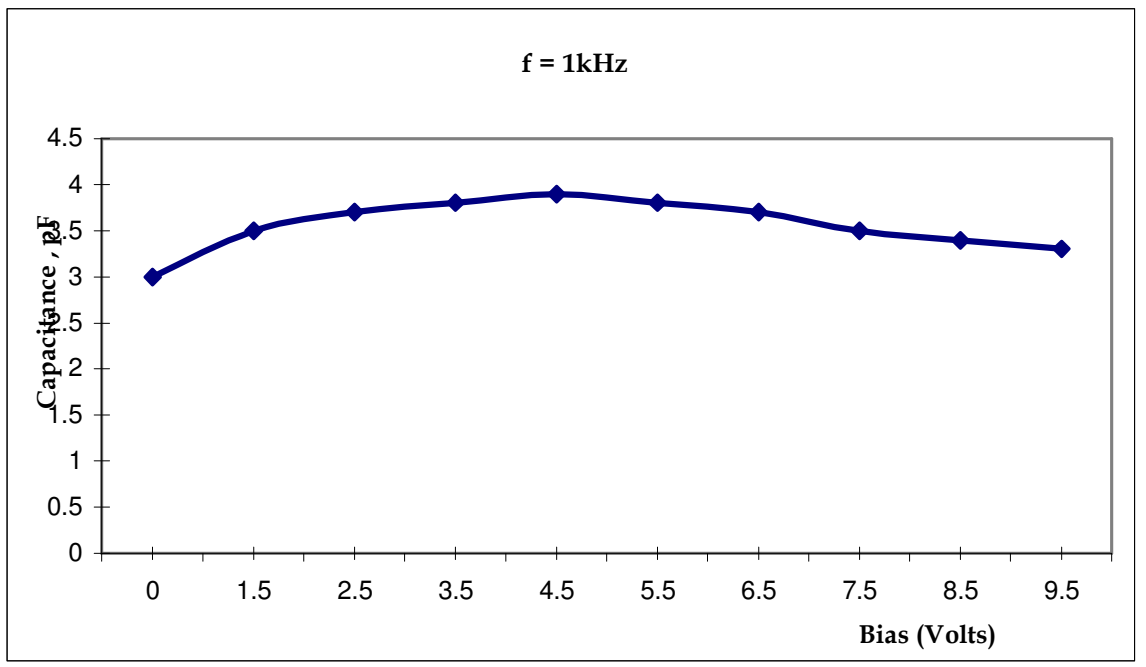

Fig. (1): Typical polarized mercuric iodide capacitance- voltage curves at $1 \mathrm{kHz}$. 


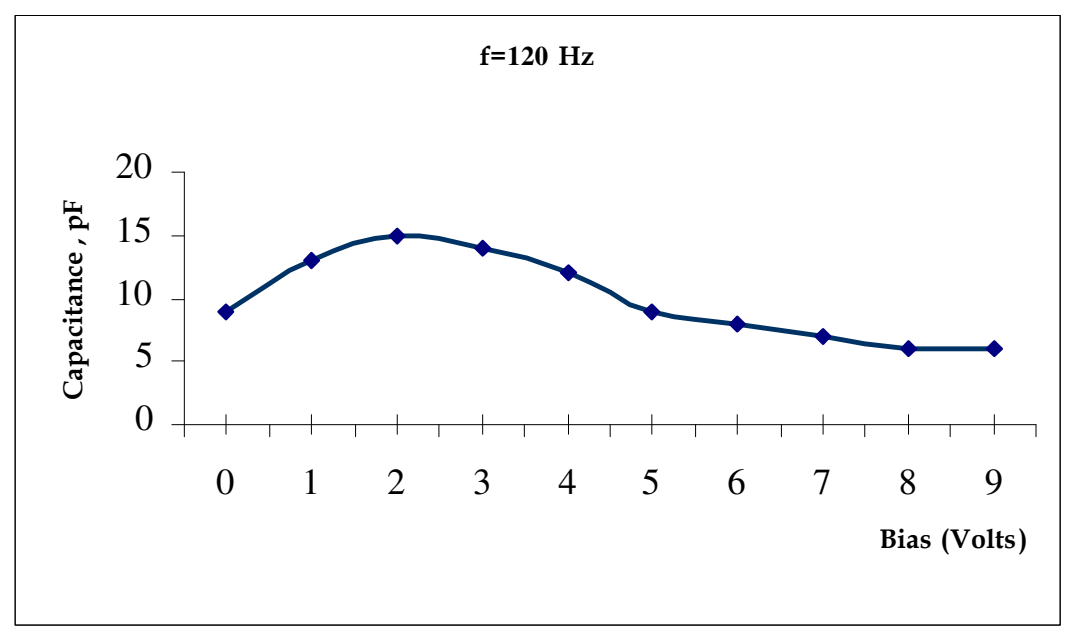

Fig. (2): Typical polarized mercuric iodide capacitance-voltage curves at $120 \mathrm{~Hz}$.

Figures ( $3 \& 4$ ) show capacitance-steady state capacitance ratio for good and polarized detectors. The steady state capacitance is the portion of the $\mathrm{C}-\mathrm{V}$ curve which shows almost constant capacitance values against variations in voltage.

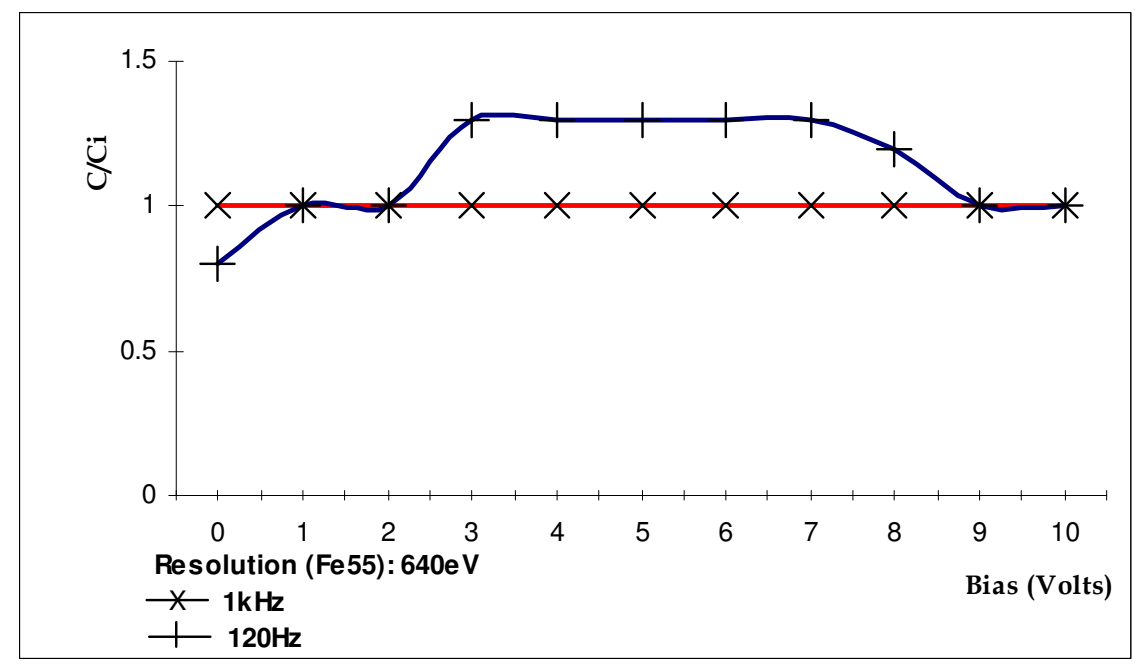

Fig. (3): Mercuric iodide detector capacitance (C)-steady state capacitance (Ci) ratio versus bias voltage for good detectors. 


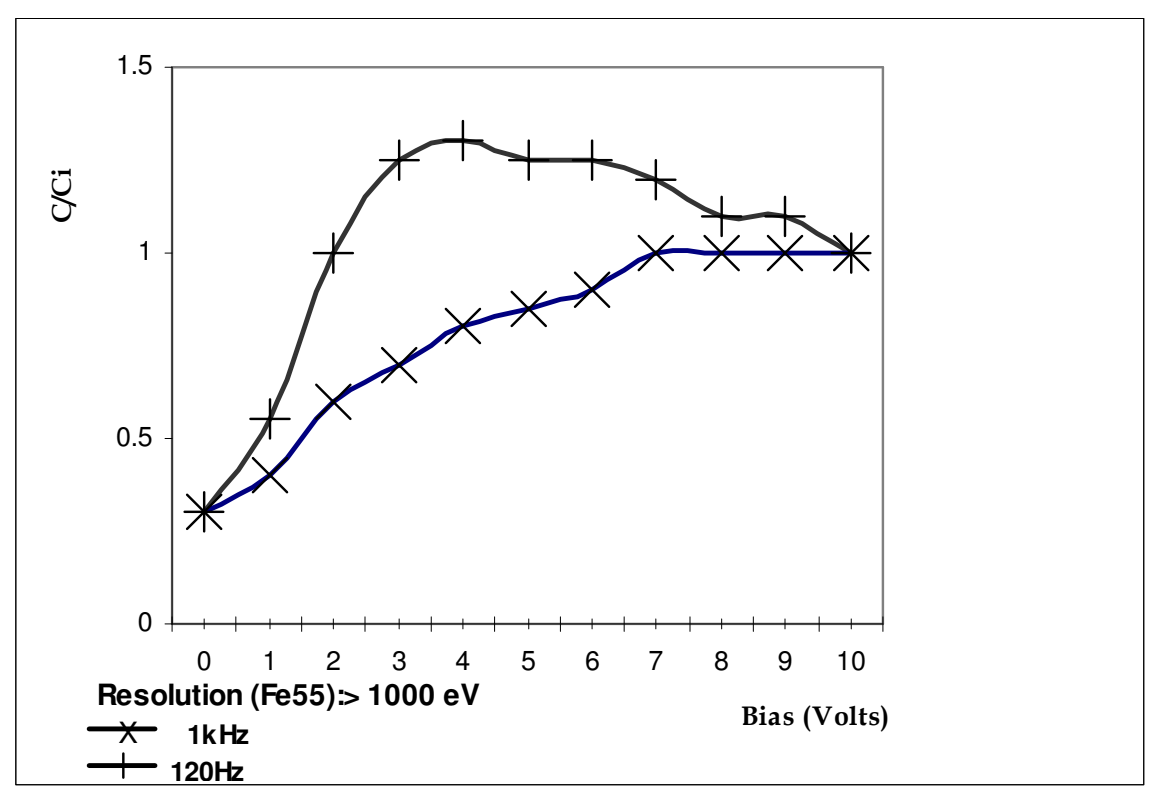

Fig. (4): Mercuric iodide detector capacitance (C)-steady state capacitance (CI) ratio versus bias voltage for polarized detectors.

\section{Discussion:}

Figures $(1 \& 2)$ confirm that mercuric iodide detectors are very close to the MIS structure because their C-V measurements exhibit a peak and the low frequency capacitance results are higher than the high frequency capacitance results [15]. Because of the above observations, we can assume that the detector capacitance is inversely proportional with the equilibrium densities of electrons (nno) for n-type detectors.

For good (un-polarized) detectors nno will be large and yields low capacitance (less than $2 \mathrm{pF}$ ) which indicates less impurities .For polarized detectors nno will be small and yields high capacitance (more than $3 \mathrm{pF}$ ) values and indicates considerable impurities and trapping centers. Also, as was indicated earlier, the capacitance variations for good quality detectors (Figure (3)) are much smaller (less than 5\%) than polarized detectors (Figure (4)). In this case we can assume that the capacitance is inversely proportional to the depletion layer width [15], i.e., good quality detectors exhibit wider depletion layers than polarized detectors. 
The above results which are based on capacitance measurements can be correlated with earlier work [8] which was based on resolution measurements. Detectors capacitance is reduced after keeping detectors in dark for more than four hours and then applying low bias voltage. Polarized spectrometers showed gradual improvement after application of bias. Also, the capacitance of polarized detectors showed a decrease after light shining. At the same time absorbed light greatly reduced the polarization in the spectrometers of the earlier work [8].

\section{Conclusion:}

$\mathrm{C}-\mathrm{V}$ measurements for mercuric iodide detectors can differentiate between good quality detectors and polarized detectors. Usually good quality detectors have less impurity and less trapping centers. As indicated MIS structure confirms the above results because theoretically the detector capacitance is inversely related to the equilibrium densities of electrons for $n$ type detectors. The mercuric iodide MIS structure with in conjunction with systematic crystal growth and detector fabrication can give information about the effects of impurities through $\mathrm{C}-\mathrm{V}$ measurements. The results could help in understanding the polarization phenomena in mercuric iodide detectors using capacitance or resolution measurements or both.

\section{References}

1. L. van den Berg and S. W. Pauly, Proc. SPIE Penetrating Radiation Systems and Applications 3769, 206(1999).

2. T. E. Schesinger and R. B. James, "Semiconductors for Room Temperature Nuclear Detector Applications", Ed. Academic Press, London, 1995.

3. E.R. Manoel, M.C. Custodio, F.E. Guimaraes, R.F. Bianchi, and A.C. Hernandes, Mat. Res 2, 75 (1999).

4. D.S. McGregor, "Horizontal Ampoule Growth and Characterization of HgI2 at Controlled Gas Pressures for X-Ray and Gamma Ray Spectrometers Report", DOE NEER Grant (2001).

5. M. Hassan, J. Crystal Growth 44, 473 (1978).

6. M. Schieber, W. Schnepple and L. van den Berg, J. Crystal Growth 33, 125 (1976).

7. J. Muheim, T. Kabayashi and E, Kaldis , Nucl, Instr . Meth. 213, 39 (1983).

8. V. Gerrish, Nuclear Instruments and Methods in Physics Research, A322, 402 (1992). 
9. V. Gerrish, "Semicoductors for Room Temperature Nuclear Detectors Applications, Simiconductors and Semimetals", T.E. Scholesinger and R.B. James, Ed. San Diego, CA, USA, Academic Press, 1995, vol. 42, pp. $510-513$.

10. T. Mohammed in Bahim, A. Frain and J. Mellet, IEEE Trans. Nuct. Sci. NS-32 (1), 581 (1985).

11. K. Zdansky, J Appl. Phys. 88, 2024 (2000).

12. J. Kemmer, D. Hauff, N. Krause, Ch. Krieglmeyer and Y. Yinxiang, Nucl. Instr. and Meth. A 439, 199 (2000).

13. J.S. Iwanczyk, J.H. Kusmiss, A.J. Dabrowski, J.B. Barton, and G.C. Huth, "Fifth Symposium of X-and Gamma-Ray Sources and Applications", University of Michigan, Ann Arbor (1981).

14. M. Hassan, Indian J. Phys. 74A, 475 (2000).

15. S. M. Sze, "Physics of Semiconductor Devices", John Wiley\& Sons, New York, 1981. 